\title{
MS37-02 | Understanding Self-Assembly of Molecular Organic Solids using NMR Crystallography: From Multicomponent Solids to Supramolecular
}

\section{HYDROGELS}

Khimyak, Yaroslav (University of East Anglia, NORWICH, GBR)

Molecular level control of the self-assembly of organic molecules at different length scales is a significant challenge both in industrial and academic research. The strength of intermolecular interactions defines physical properties of such materials, which in turn may affect bioavailability. NMR crystallography has been successful in facilitating structural analysis of organic molecular solids. However, considerable challenges remain when applying structural and dynamic methods for multi-component molecular solids across different length and time scales.

Directing molecular aggregation of pharmaceuticals can be achieved by their encapsulation inside nanosize crystallisation chambers [1, 2]. Using advanced NMR methods, we have gained unique insight into self-assembly of multicomponent organic materials within the porous solids and identified different structural environments and mobility regimes from confined crystals to liquid-like species.

We have used single and multi-component hydrogels of $I$-phenylalanine and its derivatives to develop an NMRbased analytical approach to elucidate mechanisms of supramolecular gelation [3, 4]. Gelation of I-Phe results in formation of "crystalline" gel phases. The combination of rheology, XRD diffraction and NMR methods across different dynamic regimes (including high-field ${ }^{19} \mathrm{~F} N \mathrm{NMR}$ ) revealed a very detailed picture of self-assembly in the mixed gelator hydrogels both in the well-ordered fibres and at the solid-solution interphases. This enabled us to gain mechanistic understanding of the interplay of gelation, crystallisation and dynamics of self-assembly.

[1] Nartowski, Khimyak Mol. Pharm, 2018, 4926

[2] Nartowski, Khimyak, Angew. Chem. Int. Ed. 2016, 8904.

[3] Nartowski, Khimyak, Cryst. Growth Des. 2017, 4100.

[4] Ramalhete, Khimyak, Chem. Eur. J. 2017, 8014 